Silva Rodríguez de San Miguel, J. A., Trujillo Flores, M. M., y Lámbarry Vilchis, F. (2013). La gestión del agua y la calidad percibida en su servicio. Revista Lebret (5). Bucaramanga, Colombia: Universidad Santo Tomás, pp.89-109

\title{
La gestión del agua y la calidad percibida del servicio*
}

\section{Water management and perceived quality in the service}

Jorge Alejandro Silva Rodríguez de San Miguel

Mara Maricela Trujillo Flores ${ }^{2}$

Fernando Lámbarry Vilchis

\begin{abstract}
Resumen
La gestión del agua y la calidad en su servicio son temas escritos en muchos libros y artículos, debido a la creciente preocupación mundial por la escasez del recurso. Sin embargo, pocos estudios tienen rigor científico. El objetivo de este artículo es integrar un modelo de gestión del agua con la calidad en su servicio con un enfoque administrativo. A partir de una investigación documental, se establecen conceptos y modelos administrativos sobre estos temas. Los resultados muestran que la integración de los modelos propuestos es factible y se sugiere mayor investigación científica en esta clase de modelos porque son escasos en la literatura.
\end{abstract}

\section{Palabras clave}

Gestión del agua, Calidad en el Servicio de Agua, Modelos de Gestión del Agua, Modelos de Calidad en el Servicio de Agua.

Códigos de Clasificación JEL: Q38, Q56

\begin{abstract}
Water management and quality in its service are topics written in a lot of books and papers, due to the growing world concern for the shortage of the resource; however, few studies have scientific rigor. The purpose of this paper is to integrate a water management model with quality in its service with an administrative approach. From documentary research, concepts and administrative models are established on these topics. The results show that the integration of the proposed models is feasible, and further scientific research in this kind of models is suggested because they are scarce in the literature.
\end{abstract}

\section{Keywords}

Water Management, Water Service Quality, Models of Water Management, Models of Water Service Quality.

* Artículo producto de la tesis doctoral en proceso, titulada "Modelo de gestión del agua potable y la calidad percibida en su servicio para la delegación Iztapalapa México".

1 Estudiante de Doctorado en Ciencias Administrativas del Instituto Politécnico Nacional IPN de México correo electrónico: j.a.silva@outlook.com

2 Doctora en Ciencias Administrativas. Docente-Investigadora Escuela Superior de Comercio y Administración ESCA Santo Tomás. Instituto Politécnico Nacional IPN-México. Correo electrónico: martruj7@hotmail.com

3 Doctor en Ciencias Administrativas. Docente-Investigador Escuela Superior de Comercio y Administración ESCA Santo Tomás. Instituto Politécnico Nacional IPN-México, Correo electrónico: flambarry@gmail.com 


\section{Introducción}

La gestión fue concebida en el siglo XIX en los trabajos de Bonnin (1812) y ha sido equiparada con acciones, procesos, tareas y actividades públicas. A lo largo del tiempo, el término no ha variado en su definición, sólo se ha adaptado a diferentes escenarios como resultado del desarrollo de la sociedad, y de acuerdo con Calderón y Castaño (2005) ha sido considerada como una parte de la administración.

La calidad en un servicio fue planteada como la diferencia entre las expectativas de los clientes y las percepciones de los servicios prestados (Parasuraman, Zeithmal y Berry, 1985), a partir de esta definición, el concepto fue modificándose sin perder mucho su esencia, como con la conceptualización de Cronin y Taylor (1992) en la que sólo consideran las percepciones de los clientes con respecto a un servicio.

Los modelos de gestión del agua han ido en aumento debido a la escasez de los recursos hídricos, es de destacarse el modelo de Hooper (2006) por lo integral de su propuesta. Los modelos de la calidad en su servicio han sido principalmente modificaciones de modelos de mercadotecnia que han surgido del modelo de Parasuraman et al. (1985) como el de Franceschini, Galetto y Turina (2010).

En la mayoría de las regiones del mundo se ha considerado que la gestión del agua sea responsabilidad del Estado y en consecuencia sea parte de la Administración Pública (World Water Assessment Programme, 2009). Asociaciones público-privadas han sido el modelo predominante, algunas funcionando como se pretende y otras con efectos combinados (Elnaboulsi, 2001; Organisation for Economic Co-operation and Development, 2009; World Water Assessment Programme, 2009).

En gran parte del mundo existe una creciente preocupación por el agua. Inquietan los problemas para lograr un abastecimiento y distribución regular con la calidad necesaria en numerosas ciudades, pueblos y zonas rurales (Comisión Nacional del agua, 2011). Los expertos opinan que la mayoría de los países tienen suficiente agua como para satisfacer las necesidades de los hogares, las industrias, el sector agrícola y el medio ambiente. Es importante mencionar que el problema es la gestión y será uno de los principales factores que limite el desarrollo sustentable durante las próximas décadas (Programa de las Naciones Unidas para el Desarrollo, 2006; Naciones Unidas, 2011).

La literatura revisada mostró la falta de un modelo que permita medir y analizar la gestión del agua, como recurso humano, y su relación con la calidad en su servicio mediante un enfoque administrativo y de una manera válida y confiable; debido al poco rigor científico de los modelos y su tendencia a análisis de factores operativos y mercadotécnicos, tal como se explica en el apartado de análisis de los modelos de gestión del agua y la calidad en su servicio del presente artículo. 
Este artículo analiza la gestión del agua como recurso humano y la calidad en su servicio mediante un enfoque administrativo, haciendo énfasis especial en el análisis de los modelos que existen sobre estos fenómenos, con la finalidad de integrar un modelo de gestión del agua con la calidad en su servicio bajo el enfoque establecido. Para alcanzar el objetivo propuesto, se cubren una serie de puntos. Primero, para establecer el marco de referencia se hace una revisión del estudio de estos temas para clarificar cómo es que se lleva a cabo la gestión y qué entidades intervienen en esto. Segundo, se define la gestión del agua y la calidad en su servicio a través de los principales autores que los han estudiado. Tercero, se analizan los modelos más importantes localizados en torno a estos temas señalándose sus ventajas y desventajas.

\section{Conceptualización de la gestión del agua}

La primera vez que se utilizó el término gestión fue a principios del siglo XIX en los trabajos de Bonnin (1812). Este autor equipara la gestión y la administración con acciones, procesos, tareas y actividades para ocuparse de cuestiones públicas. Posteriormente, Fremont y Rosenzweig (1979) tienen un enfoque hacia el sector privado y señalan que la gestión debe abarcar la optimización a través de la dirección tecnológica, la organización de la gente y la relación de la organización con su entorno. De Bruyne (1983) menciona que la gestión comprende un saber y una práctica que apela a la ciencia y al arte, es una definición general que se enfoca en el pragmatismo. En cambio, la definición de Aubert y Gaulejac (1987) complementa la anterior porque asevera que la función fundamental de la gestión es producir organización en el sentido de poner en marcha mecanismos que permitan resolver los conflictos que aparecen día con día dentro de una organización y conseguir una convivencia relativamente ordenada de elementos donde exista tensión continua. Martínez y Camargo (1995) continúan en la misma línea que los autores que la definen con un enfoque en los negocios, indican que la gestión se refiere a objetivos de desarrollo social, a la administración de las organizaciones en interacción con la racionalidad social del entorno. Brugué y Subirats (1996) plantean que gestión significa utilizar el conocimiento como mecanismo para facilitar una mejora continua, asumir la responsabilidad sobre la acción de un sistema.

Otros autores como Pacheco, Castañeda y Caicedo (2002) complementan la definición mencionada en los párrafos anteriores, porque consideran a la gestión como un sistema que incluye objetivos, estrategia, tecnología, estructura y procesos organizativos, cultura empresarial y personalidad de los miembros de una organización. Calderón y Castaño (2005) mencionan que se ha establecido que el objeto de estudio de la administración es la gestión y el gobierno de la gente en una organización, lo cual recae en el sector público, tal como el enfoque que da Sánchez (2003), quien explica la gestión como la dinámica operacional de las instituciones que orienta el trabajo de sus funcionarios hacia el logro de resultados. Detalla que 
la gestión es la razón de ser del Poder Ejecutivo de un país, ya que se encamina a asuntos de interés público que los ciudadanos de una entidad reconocen como propios. Drucker (2007) señala que la gestión debe concebirse en la interpretación de lo que él denomina la "teoría del negocio", teniendo como propósito facultar a una organización para alcanzar sus resultados en un medio imprevisible.

Como se observa, los conceptos de gestión son explicados por diversos autores bajo diferentes perspectivas y hay diferencias en la definición del concepto entre el sector público y el sector privado. La definición desde la perspectiva del sector público es más amplia, ya que considera al gobierno de un país en su intervención (Sánchez, 2003), porque surgió como complemento al estudio de las políticas públicas (Bozeman, 1993).

Las definiciones enunciadas en seguida se enfocan en la gestión del agua y se relacionan con la mayoría de los conceptos de gestión que se han detallado, en el sentido de que integran a diversos actores que intervienen en ésta, aunado a que se le da énfasis a la parte activa de este fenómeno. Es importante resaltar que existen distintos enfoques para asumir la gestión del agua, desde enfoques rurales pensados para el abastecimiento de agua en esas zonas, otros basados en la unidad de cuenca, hasta enfoques urbanos de abastecimiento de agua potable. La diferencia estriba en la participación de un mayor número de organizaciones separadas en las zonas rurales y mayor centralización de éstas en las zonas urbanas (Santos, Cordery y Lacovides, 2009).

Para Mollinga (1998), la gestión del agua es un modo de interacción social de diversos actores, para lo cual se emplean diferentes métodos, recursos y estrategias en torno a actividades de uso y distribución de agua, las cuales toman lugar en un determinado sistema socio técnico que radica de un conjunto de espacios de interacción que tienen una dimensión espacial en forma de niveles sociales hidráulicos del sistema de riego (sistema, grupo de familias, familia) y una dimensión de tiempo vinculado con el ciclo agroecológico y el ritmo de la entrega de agua. Está enraizada en la cultura, en la estructura agraria, en la infraestructura institucional de entidades públicas y privadas y en la infraestructura material (ecológica y tecnología), las que son reproducidas consecutivamente y trasmutadas a través de interacción. Martínez, Graf, Santana y García (2005) explican la gestión del agua como una serie de acciones desarrolladas de manera integral por los actores de una cuenca para la conservación, manejo, suministro y disposición del agua en una cuenca, que garanticen la calidad y cantidad para el desarrollo sostenible de la sociedad. La Ley de Aguas Nacionales (2012) de México señala que la gestión del agua comprende en su totalidad a la administración gubernamental del agua. Se define como un proceso sostenido en una serie de principios, políticas, actos, recursos, instrumentos, normas formales y no formales, bienes, recursos, derechos, atribuciones y responsabilidades, a través del cual coordinadamente el Estado, los usuarios del agua y las organizaciones de la sociedad, fomentan e instrumentan para lograr el desarrollo sustentable en beneficio 
de los seres humanos y su medio social, económico y ambiental. La definición anterior es muy vasta porque considera a diferentes entes y actividades dentro de la gestión mediante un enfoque hacia el sector público. Y aquí se pregunta: ¿Cómo se ha conceptualizado la calidad en un servicio y el uso de este elemento? A continuación se explica brevemente.

\section{Conceptualización de la calidad en un servicio}

En la literatura de mercadotecnia, bienes y servicios son ambos descritos como productos (Cowell, 1991). En otras palabras, los productos intangibles son referidos a menudo como servicios. Los servicios son un tipo diferente de producto de bienes para satisfacer las necesidades de un cliente (Foxall, 1985). Algunos productos son una mezcla de un servicio tangible e intangible (Kandampully, Mok y Sparks, 2013).

La definición conceptual del término calidad del servicio se puede analizar desde tres perspectivas: a) como la comparación entre expectativas y las percepciones de los clientes; b) como la actitud o satisfacción de los clientes por los servicios de un proveedor; c) como una estrategia competitiva de diferenciación.

a) Dentro del primer rubro, se encuentra que la calidad es lo que los clientes perciben, y esto se da por medio de un producto tangible o intangible en un servicio (Grönroos, 1994) también se puede explicar mediante un juicio por parte de los clientes respecto a lo que buscan encontrar en un servicio (Devlin y Dong, 1994). El conocimiento que se tenga de la calidad de los productos tangibles no es suficiente para entender la calidad de los servicios, porque ambos son distintos, dada la existencia de un conjunto de características de los servicios que los diferencian de los productos tangibles (Parasuraman, Zeithmal y Berry, 1985). La definición de Parasuraman, Zeithaml y Berry (1988) es la más generalizada, estos autores definen la calidad de un servicio como la diferencia entre las expectativas de los clientes y sus percepciones de los servicios prestados por empresas de ese giro. Nitecki y Hernon (2000) conceptualizan la calidad del servicio en términos de cumplir o superar las expectativas del cliente o la diferencia entre las percepciones del cliente y las expectativas del servicio, lo cual no difiere tanto de la definición anterior. Con Oliver (1980), surgió el paradigma de la disconformidad, que consiste en valorar la calidad de un servicio percibido como una desavenencia entre expectativas y percepciones. Si el servicio recibido por el cliente está dentro de una serie de parámetros de tolerancia o supera el nivel deseado, la organización que presta el servicio se encuentra en un contexto de ventaja competitiva, o viceversa (Zeithaml y Bitner, 2002). Cronin y Taylor (1992) y Teas (1993) indican que es posible definir la calidad de un servicio sólo con la consideración de las percepciones que existan de los clientes en la prestación 
de un servicio, lo cual contrasta con las definiciones de Nitecki y Hernon (2000) y Parasuraman et al. (1988).

b) En el segundo ámbito de la definición, Parasuraman et al. (1988) indican que la calidad de un servicio es el grado en que un servicio satisface o excede las expectativas de los clientes. La calidad de un servicio incluye la satisfacción del cliente, la repetición de compra y la recomendación posterior, todo esto puede originar lealtad a un proveedor (Olshavsky y Rosen, 1985; Cronin y Taylor, 1992; Teas, 1993; Oh, 1999). La satisfacción está fundamentada en la experiencia con un servicio, en tanto que la calidad percibida del servicio no está basada necesariamente en eso. Por ello, la investigación sobre la satisfacción se ha centrado en evaluaciones después del consumo, mientras que la de calidad del servicio en algunos casos ha focalizado su atención en evaluaciones antes del consumo (Latour y Peat, 1979). De acuerdo con esta perspectiva, Hunt (1977) explica que la satisfacción de un consumidor es el carácter favorable de la evaluación subjetiva de un individuo, de los diversos resultados y experiencias asociadas con la compra o utilización de un producto. Además, puede entenderse como una evaluación de una emoción inherente en la adquisición de un producto o experiencia del consumo (Oliver, 1980) o como un juicio evaluativo posterior a la compra (Day, 1984), en estas definiciones destaca el aspecto emotivo. Se ha señalado que la satisfacción es diferente de las creencias cognitivas sobre los resultados de un producto o el consumo (Westbrook, 1987). Es por ello que Halstead, Droge y Cooper (1993) detallan que la satisfacción del cliente se da por una respuesta afectiva vinculada a una transacción específica que deriva de una comparación del resultado de un producto con algún estándar fijado con anterioridad a la compra. Fornell (1992) y Oliver (1997) consideran que la etapa de su definición es posterior al consumo y es la comparación del resultado percibido después de la compra con las expectativas previas a ésta. A su vez, Berné (1997) la define como el sentimiento que describe la experiencia de consumo total acumulado de los clientes con el servicio, lo cual es una evaluación completa de la experiencia de compra y consumo. Esta última definición complementa a las demás, por integrar un proceso completo de la utilización de un servicio que pueda generar satisfacción.

c) En la tercera perspectiva, se plantea que la entrega de altos niveles de calidad de un servicio es una estrategia para el éxito y la supervivencia para las empresas, alineada a la creación de ventajas competitivas de diferenciación frente a la competencia (Porter, 1985; Brown y Swartz, 1989; Santos, 2003). Bitner, Stephen y Matthew (2000) señalan que la calidad de un servicio es el impacto o la impresión total que tienen los clientes sobre la inferioridad o superioridad de una empresa y sus servicios respecto a la competencia. Con estas definiciones, se integra la competitividad de empresas de servicios 
como elemento para que se tenga confianza de que un consumidor recibirá un servicio de calidad.

Como se observa, las conceptualizaciones de los dos términos establecidos como referencia en el estudio son variadas y van cambiando de acuerdo a los contextos y épocas en los que fueron concebidos, pero algo muy importante son las acciones con que se ha logrado la gestión del elemento a fin de brindar un servicio con calidad.

\section{Método de investigación}

Se llevó a cabo una investigación documental, la cual consistió en una revisión bibliográfica que permitió el análisis de libros, tesis de maestría y doctorado, documentos técnicos, páginas de internet y artículos científicos de diferentes bases de datos entre las que destacan las siguientes: JSTOR, EBSCO, Springer, Elsevier, entre otras. Se buscaron modelos de gestión del agua que tuvieran un enfoque administrativo, se encontraron 12 modelos principales, los cuales se seleccionaron tomando como criterio el índice de citación y, adicionalmente, que el modelo contara con evidencia bajo métodos formales. En el caso de la calidad del servicio de agua, se realizó el mismo procedimiento, se identificaron 26 modelos principales y 2 adaptaciones del modelo SERVQUAL ${ }^{4}$ (Parasuraman et al., 1988).

\section{Análisis de los modelos de gestión del agua y su calidad}

En la búsqueda de información, se evidenció un vacío de modelos de gestión del agua, ya que la gran mayoría de ellos no permite la medición de la gestión del agua, únicamente propone un conjunto de variables y dimensiones pero no cuenta con instrumentos confiables y de validez científica, como se profundizará en el apartado correspondiente. Otro punto observado fue el hecho de que existen modelos que se enfocan fundamentalmente al aspecto operativo, y otros al administrativo, como los de Saleth y Dinar (1999), Hooper (2006) y el del Consultivo del Agua (2011). Es importante recalcar que varios modelos son adaptados de otras disciplinas o se pueden adaptar para la gestión del agua, por ejemplo el cuadro de mando integral de Kaplan y Norton (1997).

Para la calidad en el servicio del agua, sólo hay adaptaciones del modelo de Parasuraman et al. (1994) y de Cronin y Taylor (1992), ya que éstos cuentan con validez y confiabilidad rigurosa, como se analizará en el apartado dedicado a este tema. Por el hecho de ser muy generales, los modelos que existen en la literatura se pueden adaptar a una disciplina en concreto (en este caso a la gestión ambiental), como el caso del modelo de Franceschini et al. (2010).

4 SERVQUAL es el acrónimo de service quality, en español significa calidad del servicio. 


\section{Modelos de gestión del agua}

Una vez hecho el análisis correspondiente, a continuación se profundiza en los modelos fundamentales localizados que se deben tener en cuenta en la gestión del agua.

\section{Gestión de instituciones formales}

Saleth y Dinar (1999) señalan ciertos indicadores a utilizar para la gestión de instituciones formales. Los indicadores de las instituciones formales Saleth y Dinar (1999) tienen un enfoque muy amplio y aplicable a la evaluación del desempeño institucional. Estos autores intentan cuantificar a través de la efectividad de los elementos de las instituciones, las interrelaciones entre estos componentes y la interrelación entre las instituciones y el desempeño del sector del agua. Al analizar la eficacia de las instituciones, Saleth y Dinar (1999) descomponen sus elementos constituyentes y los elementos constituyentes adicionales en los aspectos de los componentes. De esta manera, se acercan a los indicadores a través de un efecto individual e interactivo de los elementos de las instituciones sobre el desempeño institucional, así como a través del impacto socio-económico, político y medio ambiental, relacionados con los recursos en los que funcionan.

Este modelo no cuenta con un instrumento de medición, sólo considera un conjunto de elementos de instituciones a través de una revisión de la literatura, conjuntamente con la experiencia del autor: la ley, la política y la administración. Las dimensiones son las siguientes: a) efectividad de la ley de aguas; b) efectividad de la política de aguas; c) el rendimiento global del sector del agua; d) efectividad en la administración del agua. Para ser capaz de evaluar la efectividad de cada componente, los autores que estamos reseñando toman en cuenta los aspectos que están dentro de cada uno y la fuerza de los vínculos hacia otros componentes. Se desarrollan los indicadores, no sólo en la base de su capacidad de reflejar el rendimiento de un componente o aspecto dado, sino también en función de la posibilidad de traducirlo en forma numérica (Saleth y Dinar, 1999).

\section{Gestión integral de recursos hídricos en cuencas hidrográficas}

En este modelo, Hooper (2006) desarrolló indicadores generales de desempeño en dos etapas: la primera consistió en la recopilación de las mejores prácticas conocidas en la gestión integrada de cuencas hidrográficas a partir de la revisión de la literatura desde la época de 1970 y la revisión de las experiencias de los profesionales, consultores, administradores de cuencas y gestores de los recursos hídricos en el campo a nivel internacional. Este material se complementó con discusiones de gente con la que Hooper (2006) interactuó en ese momento, y la cual está involucrada en proyectos de consultoría en gestión de cuencas. 
La segunda etapa consistió en la síntesis de las mejores prácticas para crear un conjunto de indicadores generales. Es decir, lo que es considerado por los profesionales en cuencas como el conjunto de prácticas de gestión que dará lugar muy probablemente a la implementación de la gestión integrada de los recursos hídricos. Este estudio capturó la esencia del significado de cada término y proporcionó una amplia variedad de indicadores (Hooper, 2006). El resultado de tan vasta investigación dio como resultado la identificación de 115 indicadores de buenas prácticas integradas en la gestión de cuencas hidrográficas (Hooper, 2006), dentro de las 10 dimensiones principales identificadas que se mencionan a continuación:

- Toma de decisiones coordinada: utilización de mecanismos de coordinación entre y dentro de agencias y organismos de cuenca, consenso basado en la toma de decisiones, vínculos entre las instituciones locales del agua y un organismo de cuenca, participación de los intereses sectoriales pertinentes.

- Respuesta en la toma de decisiones: procesos de decisión que se adapten a nuevo conocimiento y a nuevas condiciones, promuevan la eficiencia, el valor del diálogo intersectorial y las mejores prácticas.

- Objetivos, cambio de objetivos y finalización del objetivo: logro de objetivos mediante un enfoque integrado.

- Sostenibilidad financiera: evidencia de apoyo financiero en curso, registro de los gastos y transparencia en el proceso.

- Diseño organizacional: utilización de procesos democráticos, evidencia de acuerdos internacionales estables, evidencia de una política hídrica nacional propicia para la gestión de cuencas hidrográficas, uso de estructuras organizacionales que se adapten a las necesidades de una cuenca y eviten la fragmentación.

- El papel de la ley: existencia de leyes que apoyan la gestión de cuencas hidrográficas, y leyes caracterizadas por arreglos fuertes y flexibles.

- Formación y desarrollo de capacidades: capacitación de personal de manera continua y pertinente para las necesidades de una cuenca.

- Información e investigación: existencia de un sistema de conocimiento para facilitar la toma de decisiones, protocolos para compartir información y una cultura de vínculos entre investigación y conocimiento.

- Rendición de cuentas y supervisión: evidencia de que organismos de cuenca son responsables ante componentes de gobiernos, ciudadanos y hacen uso de mecanismos de información transparentes.

- Funciones del sector público y privado: evidencia de participación de las partes interesadas: especificación clara de funciones del sector público y privado. 
Hooper (2006) también elaboró un instrumento para la medición de los indicadores, para ello se utilizó la escala tipo Likert. Este modelo ha sido aplicado en los Estados Unidos de América. Sin embargo, no se señala una validez y confiabilidad estadística del instrumento.

\section{Gestión del agua del Consejo Consultivo de México}

Este modelo se puede aplicar en organizaciones de otros países en los cuales el sector público gestione los recursos hídricos, por lo genérico que es, a pesar de que fue diseñado para el contexto de México en el año 2010 por el Consejo Consultivo del Agua (2011). Fue un ejercicio novedoso y asumió riesgos relevantes, ya que no existía una manera factible de evaluar y comparar integralmente el desempeño en materia de gestión hídrica. Se enviaron cuestionarios a organismos de sistemas de agua potable, alcantarillado y saneamiento en 50 ciudades de México, en los cuales se solicitó información considerada importante para representar a las dimensiones seleccionadas. Se pretendió cubrir un universo de ciudades con población atendida superior a 250 mil habitantes e incluyó, además, a todas las capitales de las entidades federativas.

En el modelo, se definen cinco dimensiones esenciales para la gestión de los sistemas de agua potable y saneamiento, las cuales se determinaron con la asesoría de expertos en el tema: la eficiencia, en ésta se consideran aspectos de tipo operativo; las finanzas públicas, que son los ingresos y egresos que tiene una organización; la calidad del servicio, que considera la continuidad del agua; el medio ambiente, que señala el tratamiento de aguas residuales y la institucionalidad, que incorpora aspectos administrativos (Consejo Consultivo del Agua, 2011). Se utilizó un instrumento para recabar información de gestores sobre el tema, pero tampoco se indica una validez y confiabilidad estadística rigurosa del instrumento empleado.

\section{Elementos comunes en los modelos en la gestión del agua}

Se encontró que Hooper (2006) incorpora dimensiones e indicadores que se adaptan a cualquier tipo de organización que gestione el agua, este modelo tiene semejanzas con el modelo de Saleth y Dinar (1999) por retomar aspectos legales, de políticas del agua y aspectos administrativos. Comparando con el modelo del Consejo Consultivo del Agua (2011), Hooper (2006) no considera la eficiencia, sólo formula indicadores que pueden dar la pauta para ello, sobre todo en el uso del tiempo de respuesta de un organismo gestor y el seguimiento de ciertos lineamientos o programas. El mismo autor considera la parte financiera, la calidad del servicio desde una perspectiva química y formula en algunos indicadores la parte de sustentabilidad medioambiental. La dimensión "institucionalidad" del Consejo Consultivo del Agua (2011) se puede corresponder con muchas de las dimensiones planteadas por el mismo autor. 
En la Tabla 1. se observa el análisis principal de los modelos de gestión del agua.

Tabla 1. Análisis de los modelos de gestión del agua

\begin{tabular}{|c|c|c|c|c|}
\hline Modelos & Dimensiones & $\begin{array}{c}\text { Área de } \\
\text { aplicación }\end{array}$ & Ventajas & Desventajas \\
\hline $\begin{array}{l}\text { Gestión de } \\
\text { instituciones } \\
\text { formales } \\
\text { (Saleth y } \\
\text { Dinar, 1999). }\end{array}$ & $\begin{array}{l}\text { Cuatro dimensiones: } \\
\text { efectividad de la Ley } \\
\text { de aguas, efectividad } \\
\text { de la política de aguas, } \\
\text { efectividad en la } \\
\text { administración del agua } \\
\text { y rendimiento global del } \\
\text { sector del agua. }\end{array}$ & $\begin{array}{l}\text { Gestión } \\
\text { ambiental. }\end{array}$ & $\begin{array}{l}\text { Es un modelo } \\
\text { orientado a la } \\
\text { efectividad y } \\
\text { es aplicable a } \\
\text { instituciones } \\
\text { formales que } \\
\text { gestionen el } \\
\text { agua. }\end{array}$ & $\begin{array}{l}\text { Es limitado a lo } \\
\text { político y no cuenta } \\
\text { con un instrumento } \\
\text { validado y } \\
\text { confiabilizado } \\
\text { estadísticamente. }\end{array}$ \\
\hline $\begin{array}{l}\text { Gestión } \\
\text { integral de } \\
\text { recursos } \\
\text { hídricos } \\
\text { en cuencas } \\
\text { hidrográficas } \\
\text { (Hooper, } \\
\text { 2006). }\end{array}$ & $\begin{array}{l}\text { Diez dimensiones: toma de } \\
\text { decisiones coordinada; } \\
\text { respuesta en la toma de } \\
\text { decisiones; objetivos, } \\
\text { cambio de objetivos y la } \\
\text { finalización del objetivo; } \\
\text { sostenibilidad financiera; } \\
\text { diseño organizacional; } \\
\text { el papel de la ley; } \\
\text { formación y desarrollo de } \\
\text { capacidades; información } \\
\text { e investigación; rendición } \\
\text { de cuentas y supervisión y } \\
\text { funciones del sector público } \\
\text { y privado. }\end{array}$ & $\begin{array}{l}\text { Gestión } \\
\text { ambiental. }\end{array}$ & $\begin{array}{l}\text { Investigación } \\
\text { exhaustiva } \\
\text { holística para la } \\
\text { identificación de } \\
115 \text { indicadores } \\
\text { aplicables a } \\
\text { organizaciones } \\
\text { de gestión de } \\
\text { agua. }\end{array}$ & $\begin{array}{l}\text { Falta que sea } \\
\text { aplicado en } \\
\text { diferentes } \\
\text { contextos. No posee } \\
\text { un instrumento } \\
\text { validado y } \\
\text { confiabilizado } \\
\text { estadísticamente. }\end{array}$ \\
\hline $\begin{array}{l}\text { Gestión del } \\
\text { agua en las } \\
\text { Ciudades } \\
\text { de México } \\
\text { (Consejo } \\
\text { Consultivo del } \\
\text { Agua, 2011). }\end{array}$ & $\begin{array}{l}\text { Cinco dimensiones: } \\
\text { eficiencia, finanzas } \\
\text { públicas, } \\
\text { calidad del servicio, } \\
\text { medio ambiente e } \\
\text { Institucionalidad. }\end{array}$ & $\begin{array}{l}\text { Gestión } \\
\text { ambiental. }\end{array}$ & $\begin{array}{l}\text { Se presentan } \\
\text { las principales } \\
\text { dimensiones } \\
\text { para una gestión } \\
\text { integral del } \\
\text { agua. }\end{array}$ & $\begin{array}{l}\text { Está limitado por } \\
\text { las dimensiones } \\
\text { utilizadas y se } \\
\text { enfoca más al } \\
\text { aspecto operativo, } \\
\text { en lugar de } \\
\text { un enfoque } \\
\text { administrativo. } \\
\text { No cuenta con } \\
\text { un instrumento } \\
\text { validado y } \\
\text { confiabilizado } \\
\text { científicamente. }\end{array}$ \\
\hline
\end{tabular}

Fuente: Autor con base en los modelos planteados por los autores mencionados en la Tabla.

\section{Modelos para la calidad en un servicio}

A continuación se describen los principales modelos utilizados para la medición de la calidad de un servicio: 


\section{Modelo SERVQUAL.}

A pesar de la importancia del estudio de los servicios por separado de los bienes, se hace evidente en la década de 1980 que nadie había estudiado seriamente qué es la calidad y cómo medirla (Schneider y White, 2004). Un programa puesto en marcha por Zeithaml, Parasuraman y Berry (1988) dio lugar al desarrollo de un modelo que acompaña la medición de la calidad en un servicio, denominado SERVQUAL, en el que destaca la comparación de las expectativas del cliente.

El Modelo SERVQUAL de calidad del servicio fue elaborado por Parasuraman et al., (1994) cuyo propósito fue mejorar la calidad del servicio ofrecida por organizaciones. Estos autores analizan en principio diez dimensiones, las cuales fueron consideradas fundamentales para la prestación de un servicio pero, tras un análisis estadístico de las diez dimensiones enunciadas, encontraron elevadas correlaciones entre varias de éstas. Por consiguiente, agruparon las diez dimensiones para obtener finalmente cinco dimensiones de la calidad del servicio: 1) fiabilidad, que es ejecutar el servicio eficazmente; 2) capacidad de respuesta, es el ofrecimiento de un servicio rápido; 3) seguridad, es la falta de riesgos; 4) empatía, se enfatiza en el respeto y la amabilidad; 5) elementos tangibles, en los que interviene la imagen de las instalaciones, los equipos, el personal, entre otros factores.

A partir de allí se elaboró un instrumento que permite cuantificar la calidad de un servicio, a partir de un modelo precedente de los mismos autores (Parasuraman et al., 1985) en el cual explican cinco diferencias o vacíos (gaps). Los autores mencionados calcularon la media y las desviaciones estándares de los items planteados en su instrumento, los coeficientes alfa por medio de las puntuaciones obtenidas como resultado de la divergencia entre percepciones y expectativas para las cinco dimensiones que fueron propuestas y así se obtuvo un valor de 0.92 de confiabilidad de la escala total por coeficientes alfa de Cronbach. Se recogieron 22 items que identifican las expectativas generales de los clientes sobre un servicio concreto y 22 items para la percepción de éste. Con la información obtenida de los cuestionarios, se calcularon las percepciones menos las expectativas para cada par de ítems y se establecieron relaciones para determinar si se habían alcanzado niveles altos o bajos de calidad en el servicio (Parasuraman et al., 1994).

La escala SERVQUAL ha tenido críticas, en primer lugar se encuentran las planteadas por Clow y Vorhies (1993), quienes argumentan que cuando las percepciones y las expectativas se miden simultáneamente es posible errar, debido a que los encuestados revelan que sus expectativas son mayores de lo que realmente eran antes de recibir el servicio. Uno de los autores más críticos de este modelo ha sido Buttle (1996), quien cuestiona la utilización de la discrepancia como fundamento para evaluar la calidad de un servicio, el número de dimensiones empleadas, el empleo de una escala tipo Likert de siete puntos, la ambigüedad que se genera por la duplicación de las preguntas para las expectativas y para las percepciones y el vocabulario utilizado en los ítems. 
Adaptación del modelo SERVQUAL en los servicios de agua.

Franceschini et al. (2010) adaptaron el modelo SERVQUAL de Parasuraman et al., (1994) a los servicios de agua, para lo cual utilizaron las 10 primeras dimensiones del estudio de estos autores: 1) fiabilidad, que es la cobertura de agua y el cumplimiento de normas y programas; 2) capacidad de respuesta, que describe la preparación que existe para el mantenimiento del sistema de agua, las citas con los clientes y la respuesta a quejas y sugerencias; 3) la competencia, que detalla las características técnicas y para el servicio al cliente; 4) el acceso, entendido como las diferentes formas en las que el cliente puede contactar a las organizaciones tomando en cuenta su disponibilidad; 5) la cortesía es la amabilidad del personal de contacto; 6) la comunicación o información existente para el sistema de agua; 7) la credibilidad en la que se retoman cuestiones de la imagen que tiene el cliente de las organizaciones de gestión del agua; 8) la seguridad, entendida como la salida de agua del grifo cuando se requiera; 9) la comprensión del cliente, que es el esfuerzo de la compañía por entender las necesidades de los clientes; 10) los elementos tangibles, que son químicos existentes en el agua que determinan su calidad, así como parte de la infraestructura que se utiliza para su suministro.

Franceschini et al., (2010) también elaboraron un instrumento de medición tomando como base el instrumento SERVQUAL, el cual emplea la escala tipo Likert. En su estudio, los autores estructuran su enfoque en dos partes: el primero es la evaluación general de la calidad del servicio, mientras que el segundo considera aspectos específicos de servicios que permiten entender mejor las posibles razones de insatisfacción. Sin embargo, la confiabilidad y validez estadística no es tan rigurosa en el instrumento de medición, ya que no se calculan coeficientes alfa de Cronbach, por ejemplo.

De igual forma, se describe una aplicación de software específico para apoyar la recolección y análisis estadístico de datos. La herramienta propuesta garantiza una evaluación global de la calidad de servicio desde el punto de vista del cliente. Su organización en dos partes permite la reducción de los costes globales de adquisición de datos, así como la maximización de la información adquirida de clientes. El instrumento puede ser adaptado con el fin de conocer necesidades de clientes y de un controlador de un servicio en zonas supervisadas (Franceschini et al., 2010).

\section{Modelo SERVPERF}

En este modelo, Cronin y Taylor (1992) investigaron la conceptualización y medición de la calidad del servicio, su relación con la satisfacción del consumidor y las intenciones de compra. Estos autores compararon las puntuaciones calculadas para concluir que las percepciones son mejores predictores de la calidad del servicio. Arguyeron, en el marco del modelo de Parasuraman et al. (1985) respecto a la conceptualización y medición de la calidad del servicio y el rendimiento de la 
medición desarrollada. A su modelo le llamaron SERVPERF, porque ilustra que la calidad del servicio es una forma de la actitud de un consumidor. Explican que el instrumento del SERVQUAL confunde la satisfacción y la actitud. Afirmaron que la calidad del servicio puede ser conceptualizada como algo similar a una actitud y puede ser operacionalizada por la adecuación de la importancia del modelo. Con la formulación de su modelo, se pretendió superar las restricciones que se originan al momento de utilizar las expectativas en la medición de la calidad, sin definir de una manera concreta qué tipo y qué nivel de expectativas son las que deben intervenir.

El instrumento SERVPERF está constituido por los mismos items y dimensiones que el SERVQUAL: está compuesto por 22 items de percepción de la escala SERVQUAL, pero no considera alguna alusión hacia las expectativas, por lo tanto, se reducen a la mitad las preguntas. El SERVPERF tiene un alto grado de confiabilidad: con el coeficiente alfa de Cronbach se obtuvo un valor de 0,9098 y con el alfa estandarizado un valor de 0,9238. A la vez, goza de una mayor validez predictiva que el SERVQUAL (Cronin y Taylor, 1992).

Los 22 ítems que se relacionan con las percepciones fueron tomados por estos autores de la escala SERVQUAL de Parasuraman et al. (1994). De los items de la escala original del SERVQUAL relacionados con las percepciones y las expectativas, Cronin y Taylor (1992) seleccionaron las ponderaciones relacionadas con el nivel de importancia y fueron adaptadas. La medida directa de la calidad del servicio, así como la satisfacción y la lealtad, estaban fundamentadas en el resultado de un asunto de diferencias a un asunto diferencial semántico de siete puntos. La escala SERVPERF arroja un puntaje acumulado de la calidad general de un servicio, por lo tanto el modelo SERVPERF enfatiza las implicaciones sobre las actitudes y la satisfacción (Cronin y Taylor, 1992).

\section{Elementos comunes en los modelos para la calidad en un servicio}

En la literatura se encontró que la mayoría de los modelos que existen son adaptaciones del modelo SERVQUAL de Parasuraman et al. (1994) ya que su instrumento es muy completo para medir la calidad en un servicio. Por ejemplo, Cronin y Taylor (1992) únicamente mejoraron el instrumento de Parasuraman et al. (1994) por lo cual retomaron las mismas dimensiones, pero quitaron todo lo que tenía que ver con las expectativas de los clientes y dejaron lo que se relaciona con las percepciones de éstos, por considerar de mayor relevancia y como única forma de medir la calidad del servicio. Dentro de estos modelos, cabe destacar el de Franceschini et al. (2010) estos autores retomaron las 10 dimensiones del primer modelo de Parasuraman et al. (1994) y las adoptaron para poder medir la calidad del servicio de agua.

En la Tabla 2 se analizan los principales modelos de calidad en un servicio a partir de sus dimensiones, el área de aplicación, ventajas y desventajas. 
Tabla 2. Análisis de los modelos de calidad en un servicio

\begin{tabular}{|c|c|c|c|c|}
\hline Modelos & Dimensiones & $\begin{array}{c}\text { Área de } \\
\text { aplicación }\end{array}$ & Ventajas & Desventajas \\
\hline $\begin{array}{l}\text { Modelo } \\
\text { SERVQUAL de } \\
\text { Parasuraman et } \\
\text { al., (1994) }\end{array}$ & $\begin{array}{l}\text { Cinco dimensiones: } \\
\text { fiabilidad, capacidad de } \\
\text { respuesta, seguridad, } \\
\text { empatía y elementos } \\
\text { tangibles }\end{array}$ & Mercadotecnia & $\begin{array}{l}\text { Es un modelo } \\
\text { muy completo } \\
\text { aplicable a } \\
\text { cualquier tipo } \\
\text { de organización } \\
\text { que ofrezca un } \\
\text { servicio }\end{array}$ & $\begin{array}{l}\text { La confusión que } \\
\text { produce la duplicación } \\
\text { de las preguntas para } \\
\text { las expectativas y } \\
\text { para las percepciones, } \\
\text { las dimensiones } \\
\text { empleadas pueden no } \\
\text { ser suficientes }\end{array}$ \\
\hline $\begin{array}{l}\text { Modelo } \\
\text { SERVPERF de } \\
\text { Cronin y Taylor } \\
(1992)\end{array}$ & $\begin{array}{l}\text { Cinco dimensiones: } \\
\text { fiabilidad, capacidad de } \\
\text { respuesta, seguridad, } \\
\text { empatía y elementos } \\
\text { tangibles }\end{array}$ & Mercadotecnia & $\begin{array}{l}\text { Se precisan las } \\
\text { deficiencias del } \\
\text { SERVQUAL } \\
\text { y se acorta el } \\
\text { instrumento } \\
\text { el cual goza } \\
\text { de una alta } \\
\text { confiabilidad y } \\
\text { validez. }\end{array}$ & $\begin{array}{l}\text { Está limitado, ya que } \\
\text { sólo es una mejora } \\
\text { y acortamiento } \\
\text { del instrumento } \\
\text { SERVQUAL }\end{array}$ \\
\hline $\begin{array}{l}\text { Adaptación } \\
\text { del modelo } \\
\text { SERVQUAL } \\
\text { en los servicios } \\
\text { de agua de } \\
\text { Franceschini et } \\
\text { al. (2010) }\end{array}$ & $\begin{array}{l}\text { Diez dimensiones: } \\
\text { fiabilidad, capacidad } \\
\text { de respuesta, } \\
\text { competencia, acceso, } \\
\text { cortesía, comunicación, } \\
\text { credibilidad, seguridad, } \\
\text { comprensión del } \\
\text { cliente y } \\
\text { elementos tangibles }\end{array}$ & $\begin{array}{l}\text { Gestión } \\
\text { ambiental }\end{array}$ & $\begin{array}{l}\text { Es un modelo } \\
\text { muy completo } \\
\text { para medir la } \\
\text { calidad de un } \\
\text { servicio de } \\
\text { agua en una } \\
\text { organización }\end{array}$ & $\begin{array}{l}\text { Es un estudio reciente } \\
\text { que requiere de una } \\
\text { mayor investigación }\end{array}$ \\
\hline
\end{tabular}

Fuente: Autores con base en los modelos planteados por los autores mencionados en la Tabla.

\section{Discusión y propuesta}

El modelo de Hooper (2006) se puede utilizar prácticamente en cualquier organización encargada de la gestión del agua, mientras que el modelo de Franceschini et al. (2010) se puede emplear en organizaciones encargadas de proporcionar servicios de agua. Se propone integrar el modelo de Hooper (2006) y el de Franceschini et al. (2010) para poder medir y analizar la gestión del agua y la calidad en un servicio de agua respectivamente, ya que después de realizar el análisis correspondiente, se encontró que ambos modelos tienen similitudes por los indicadores de tipo administrativo que manejan. Sin embargo, el modelo de Hooper (2006) está enfocado a los gestores, no a los usuarios que usan un servicio de agua, mientras el de Franceschini et al. (2010) se concentra en esa parte. Las dimensiones de Hooper (2006) pueden ser comparadas con varias de las planteadas por Franceschini et al. (2010). El criterio para elegir las dimensiones va a depender de la investigación a realizar y de su contexto. En este caso, se propone integrar las dimensiones mostradas en la Tabla 3 respectivamente, para cumplir con el objetivo enunciado en el presente artículo. 
Tabla 3. Propuesta de un modelo de gestión del agua y la calidad en su servicio

\begin{tabular}{|l|l|}
\hline $\begin{array}{l}\text { Dimensiones seleccionadas del modelo de } \\
\text { Hooper (2006) }\end{array}$ & $\begin{array}{l}\text { Dimensiones seleccionadas del modelo de } \\
\text { Franceschini et al. (2010) }\end{array}$ \\
\hline Toma de decisiones coordinada & Competencia \\
\hline Respuesta en la toma de decisiones & Comunicación \\
\hline $\begin{array}{l}\text { Objetivos, cambio de objetivos y la finalización } \\
\text { del objetivo }\end{array}$ & Fiabilidad \\
\hline Diseño organizacional & Acceso \\
\hline Formación y desarrollo de capacidades & Capacidad de respuesta \\
\hline Información e investigación & Credibilidad \\
\hline Rendición de cuentas y supervisión & Comprensión del cliente \\
\hline
\end{tabular}

Fuente: Autores.

En seguida se explican las relaciones entre las dimensiones del modelo:

a) La toma de decisiones coordinada se puede analizar con la competencia para observar cómo la coordinación de la toma de decisiones entre varios gestores influye en el uso equilibrado del agua, por ejemplo el agua destinada al riego, al consumo humano o al uso industrial, etc.

b) La respuesta en la toma de decisiones se puede equiparar con la comunicación para analizar si realmente los gestores toman en cuenta las opiniones de los clientes.

c) Los objetivos, cambio de objetivos y la finalización de los objetivos se tiene que ver reflejada en la fiabilidad, para observar si existe un abastecimiento constante de agua en alguna zona, con la infraestructura y el cumplimiento de normas para tal efecto.

d) El diseño organizacional es compatible con el acceso, ya que entre más compleja sea una organización, más difícil un cliente podría saber a quién dirigirse y la facilidad con la que lo haría.

e) La formación y desarrollo de capacidades se debe ver reflejada en la capacidad de repuesta, es decir, que el cliente no sufra con el abastecimiento de agua por motivos de que el personal operativo encargado de arreglar problemas en el sistema de aguas sea ineficaz. 
f) La información e investigación debe permitir la existencia de nuevos proyectos para gestionar el agua y que los usuarios de un servicio de agua tengan credibilidad en esto, debido a antecedentes positivos y a una comunicación recíproca.

g) En la rendición de cuentas y supervisión, se debe dar evidencia de que los organismos gestores del agua atiendan las necesidades de los clientes en tiempo y forma y sin actos corruptos.

\section{Conclusiones}

La gestión del agua y la calidad en su servicio han presentado cambios de acuerdo con la época y el contexto donde se les ha definido. Como sucedió en el siglo XIX en los trabajos de Bonnin (1812), que equiparó la gestión y la administración con acciones, procesos, tareas y actividades para ocuparse de cuestiones públicas y el desarrollo del concepto de la gestión del agua de Mollinga (1998), el cual es muy amplio y el más adecuado para comprender este fenómeno en la presente investigación, ya que abarca todo el proceso de la gestión porque considera muchos actores intervinientes. Los modelos analizados como el de Saleth y Dinar (1999), Hooper (2006) y el del Consultivo del Agua (2011) combinan aspectos operativos y administrativos, siendo el más completo es el de Hooper (2006), aunque la confiabilidad y validez estadística no es rigurosa.

Es de notarse que el que inició el estudio formal de la calidad en el servicio fue Parasuraman et al. (1985) de ahí la mayoría de sus sucesores solamente han modificado su concepto original. Este fenómeno fue analizado desde la perspectiva de la comparación entre expectativas y las percepciones de los clientes (Parasuraman et al., 1985), como la actitud o satisfacción de los clientes por los servicios de un proveedor (Cronin y Taylor, 1992) y como una estrategia competitiva de diferenciación (Porter, 1985).

Dentro de los modelos destaca el eliminar de Parasuraman et al. (1994) denominado SERVQUAL, en el que se hace una comparación de las expectativas del cliente. La adaptación de Franceschini et al. (2010) fue el modelo más completo localizado en los servicios de agua, pero no tiene confiabilidad y validez estadística rigurosa.

Se encontró, sin embargo, que no existen los suficientes modelos para estos fenómenos desde la perspectiva administrativa, aun a pesar de que existen algunas adaptaciones de modelos de otras disciplinas para el agua.

Con estos hallazgos y vacíos, se planteó una propuesta de integrar el modelo de Hooper (2006) junto con el de Franceschini et al. (2010). La elección de dimensiones e indicadores de estos modelos está determinada por el contexto al cual se aplique, aquí se utilizaron siete dimensiones generales de los modelos mencionados anteriormente 
y se integraron las que tienen elementos comunes, lo cual cumple con el objetivo establecido en este artículo. Esta integración de un modelo de gestión del agua con un modelo de la calidad de su servicio, pretende servir de motivación para que sea ampliado y aplicado a un contexto específico en próximas investigaciones.

Es necesario incrementar la investigación sobre la gestión del agua y la calidad en su servicio en la ciencia administrativa, ya que las investigaciones científicas serias en estos temas no son abundantes, como se analizó en este artículo. Otro punto importante es el de contribuir al campo de la gestión ambiental para tener un desarrollo sustentable en las próximas décadas en el mundo.

\section{Referencias}

Aubert, N. y Gaulejac, V. (1987). El coste de la excelencia. Barcelona, España: Paidós.

Berné, C. (1997). Modelización de la post-compra, satisfacción y lealtad. Barcelona, España: Múgica y Ruiz.

Bitner, M.J., Stephen, W.B. y Matthew, L.M. (2000). Technology infusion in service encounters. Journal of the Academy of Marketing Science, 28(1), 138-149.doi: $10.1177 / 0092070300281013$

Bonnin, C. (1812). Principes d'administration publique principes d'administration publique (3 ${ }^{e}$. éd., Vol. I). Paris, France: Chez Renaudiere.

Bozeman, B. (1993). Public management: The state of the art. San Fracisco, California: Jossey-Bass.

Brown, S.W. y Swartz, T.A. (1989). A gap analysis of professional service quality. Journal of Marketing, 53(2), 92-101. doi: 10.2307/1251416

Buttle, F. (1996). SERVQUAL: Review, critique, research agenda. European Journal of Marketing, 30(1), 8-32. doi: 10.1108/03090569610105762

Calderón, G. y Castaño, G.A. (2005). Investigación en Administración en América Latina: Evolución y Resultados. Manizales, Colombia: Universidad Nacional de Colombia.

Consejo Consultivo del Agua (2011). La gestión del agua en las ciudades de México. (Documento técnico del Consejo Consultivo del Agua). Ciudad de México, México: Consejo Consultivo del Agua.

Cowell, D. (1991). Marketing services. En Baker, M.J. (Ed.), The Marketing Book (pp. 456-466). Oxford: Butterworth Heinemann.

Cronin, J.J. y Taylor, S.A. (1992). Measuring service quality: a reexamination and extension. Journal of Marketing, 56(3), 55-68. doi: 10.2307/1252296 
Day, R. (1984). Modeling choices among alternative responses to dissatisfaction. Advances in Consumer Research, 11, 496-499.

De Bruyne, P. (1983). Teoría moderna de la administración de empresas. Madrid, España: Aguilar.

Drucker, P. (2007). Managing in the next society ( $2^{\text {nd }}$ Ed.). Great Britain: Elsevier

Elnaboulsi, J.C. (2001). Organization, management and delegation in the french water industry. Annals of Public and Cooperative Economics, 72(4), 507-547. doi: 10.1111/1467-8292.00180

Fornell, C. (1992). A national customer satisfaction parameters: The Swedish experience. Journal of Marketing, 56(1), 6-21. doi: 10.2307/1252129

Foxall, G. (1985). Marketing in the service industries. London, United Kingdom: Frank Cass.

Franceschini, F., Galetto, M. y Turina, E. (2010). Water and sewage service quality: a proposal of a new multi-questionnaire monitoring tool. Journal of Water Resources Management, 24(12), 3033-3050. doi: 10.1007/s11269-010-9593-0

Fremont, E.K. y Rosenzweig, J. (1979). Organization and management: a systems and contingency approach. United Kingdom, Dunfermline: McGraw-Hill.

Grönroos, C. (1994). Marketing y gestión de servicios: la gestión de los momentos de la verdad y la competencia en los servicios. Madrid: Editorial Díaz de Santos.

Halstead, D., Droge, C. y Cooper, M.B. (1993). Product warranties and post-purchase service: A model of consumer satisfaction. The Journal of Services Marketing, 7(1), 33-40. doi: 10.1108/08876049310026088

Hooper, B.P. (2006). Key performance indicators of river basin organizations. (World Bank Report VSP-01). Washington, EE.UU. US Army Corps of Engineering/ IWR.

Hunt, H.K. (1977). CS/D overview and future research directions. En Hunt, H.K. [eds.], Conceptualization and measurement of consumer satisfaction and dissatisfaction (pp. 455-488). Cambridge, United Kingdom: Marketing Science Institute.

Kandampully, J., Mok, C. y Sparks, B.A. (2013). Service quality management in Hospitality Tourism and Leisure. New York, EE.UU: The Haworth Press.

Kaplan, R. y Norton, D. (1997). Cuadro de mando integral. Barcelona, España: Gestión 2000.

Latour, S. y Peat, N. (1979). Conceptual and methodological issues in consumer satisfaction research. Advances in Consumer Research, 6(1), 431-437. 
Ley de Aguas Nacionales. (2012). Ciudad de México: Diario Oficial de la Federación. Consultado en febrero 18 de 2013, del sitio Web de la Cámara de Diputados: http://www.diputados.gob.mx/LeyesBiblio/pdf/16.pdf

Martínez, C.E. y Camargo, B.A. (1995). Organización y gestión de empresas: técnicas modernas de gerencia. Bogotá, Colombia: Universidad Nacional de Colombia.

Martínez, L.M., Graf, S., Santana, E. y García, S. (2005). Iniciativa intermunicipal para la gestión de la Cuenca del río Ayuquila. Veracruz, México: Universidad Veracruzana.

Mollinga, P. (1998). On the waterfront. Wather distribution, technology and agrarian change in a south indian canal irrigation system (Tesis de Doctorado). Universidad Agraria de Wageningen, Wageningen.

Naciones Unidas. (2011). Objetivos de desarrollo del milenio: Informe 2011. New York, EE.UU. Paris, France: OECD.

Nitecki, D.A. y Hernon, P. (2000). Measuring service quality at Yale University's libraries. Journal of Academic Librarianship, 26(4), 259-273. doi: 10.1016/S00991333(00)00117-8

Oh, H. (1999). Service quality, customer satisfaction and customer value: a holistic Perspective. International Journal of Hospitality Management, 18(1), 67-82. doi: 10.1016/S0278-4319(98)00047-4

Oliver, R. L. (1980). A cognitive model of the antecedents and consequences of satisfaction decisions. Journal of Marketing Research, 17(3), 460-469. doi: $10.2307 / 3150499$

Oliver, R. (1997). Satisfaction. A behavioural perspective on the consumer. New York, EE.UU.: Mc Graw-Hill.

Olshavsky, R.W. y Rosen, D.L. (1985). Use of product-testing organizations recommendations as a strategy for choice simplification. The Journal of Consumer Affairs, 19(1), 118-139. doi: 10.1111/j.1745-6606.1985.tb00347.x

Organisation for Economic Co-operation and Development (2009). Managing Water for All. Paris, France: OECD.

Pacheco, J.C., Castañeda, W.H. y Caicedo, C.H. (2002). Indicadores integrales de gestión. Bogotá, Colombia: McGraw-Hill.

Parasuraman, A., Zeithmal, V. y Berry, L. (1985). A conceptual model of service quality and its implieations for future research. Journal of Marketing, 49(4), 4150. doi:10.2307/1251430 
Parasuraman, A., Zeithhaml, V. y Berry, L. (1988). SERVQUAL; a multiple-item scale for measuring consumer perceptions of services quality. Journal of Retailing, 64(1), 12-40.

Parasuraman, A., Zeithaml, V. y Berry, L. (1994). Reassessment of expectations As a comparison standard in measuring service quality: Implications for future research. Journal of Marketing, 58(1), 111-124. doi: 10.2307/1252255

Porter, M.E. (1985). Competitive Advantage: Creating and Sustaining Superior Performance. New York, EE.UU.: Free Press.

Programa de las Naciones Unidas para el Desarrollo. (2006). Informe sobre Desarrollo Humano 2006 (euroscript Luxembourg S.à r.l., Trad.). Ciudad de México, México: Mundi-Prensa México. (Trabajo original publicado en 2006)

Sánchez, F. (2003). Planificación estratégica y gestión pública por objetivos. Serie Gestión Pública, 32, 1-80. Recuperado de http://www.eclac.cl/publicaciones/ $\mathrm{xml} / 7 / 12097 / \mathrm{sgp} 32 . \mathrm{pdf}$

Santos, J. (2003). E-service quality: A model of virtual service quality dimensions. Managing Service Quality, 13(3), 233-246. doi: 10.1108/09604520310476490

Santos, P., Cordery, I., y Lacovides, I. (2009). Coping with water scarcity: addressing the challenges [Springer version]. doi: 10.1007/978-1-4020-9579-5

Saleth, R.M. y Dinar, A. (1999). Evaluating water institutions and water sector performance. (World Bank Technical Paper No. 447). Washington, EE.UU.: The World Bank.

Schneider, B. y White, S. (2004). Service quality: research perspectives. California, EE.UU.: Sage Publications.

Teas, K.R. (1993). Expectations, performance evaluation, and consumers perceptions of quality. Journal of Marketing, 57(4), 18-34. doi: 10.2307/1252216

Westbrook, R.A. (1987). Product/Consumption (based affective responses and post purchase processes. Journal of Marketing Research, 24(3), 258-270. doi: $10.2307 / 3151636$

World Water Assessment Programme. (2009). The United Nations World Water Development report 3: water in a changing world. Paris, France: UNESCO, and London, United Kingdom: Earthscan.

Zeithaml, V., Berry, L. y Parasuraman, A. (1988). Communication and control processes in the delivery of service quality. Journal of Marketing, 52(2), 35-48. doi: $10.2307 / 1251263$

Zeithaml, V. y Bitner, M.J. (2002). Marketing de servicio: un enfoque de integración del cliente a la empresa (2da. ed.). México: McGraw-Hill. 